\title{
Formulation and Manufacturing of Solid Dosage Forms
}

\author{
Qi Tony Zhou' (D) - Tonglei Li'
}

Received: 20 November 2018 / Accepted: 21 November 2018 / Published online: 27 November 2018

(C) Springer Science+Business Media, LLC, part of Springer Nature 2018

As solid dosage forms remains the primary option when developing a drug product, it was felt by the guest editors that the need to have a special issue on formulation and manufacturing of solid products could not be overstated. We have seen the science in the field advancing from understanding of organic crystals to handling and processing to amorphous materials and to engineering and manufacturing of fine particles. Understandings at different scales and among different components in a drug product have forged into a new stage of study that calls for deeper exploration that could bridge the molecular dynamics and kinetics with product properties and in vivo performance. This special issue is hoped to showcase a few studies at this frontier.

The majority of drugs in the pharmaceutical solid products are present in the crystalline form. We have several papers that touch upon the impact of formulation and manufacturing process and storage conditions on stability and dissolution of crystalline solids. It is critical to understand such impacts for the purpose of ensuring the quality of pharmaceutical products. In this issue, Xu et al. (1) determined the effects of process conditions on the polymorphic transformation of indomethacin during hot melt extrusion granulation. In another study, Koranne et al. (2) examined impacts of formulation and lyophilization conditions on the disproportionation of indomethacin sodium, and found that $\mathrm{pH}$ shift caused by changing buffer components can lead to disproportionation and prolonged reconstitution

\footnotetext{
Qi Tony Zhou

tonyzhou@purdue.edu

Tonglei Li

tonglei@purdue.edu
}

Department of Industrial and Physical Pharmacy, College of Pharmacy, Purdue University, 575 Stadium Mall Drive, West Lafayette, Indiana 47907, USA time. Tinmanee et al. (3) established a kinetic model to quantify the effects of temperature, humidity and excipients on physical and chemical transition of Gabapentin in binary excipient mixtures after milling-induced stress. Such model may enable better understanding in the relationship between formulation and storage conditions and the stabilities of stressed pharmaceutical solids (3). In addition, we have a review article that discusses the influence of crystal anisotropy on the pharmaceutical process development such as milling, granulation, and tableting (4). The review illustrates both fundamental concepts of intermolecular and interfacial phenomena and their relevance to the pharmaceutical products, shedding light on rational design of crystalline solid formulations.

In the past decade, a large number of new drug candidates bear poor water solubility, which generally leads to poor dissolution and low bioavailability. Yamashita and Sun reported that using excess conformer (glutaric acid), the dissolution rate of carbamazepine-glutaric acid cocrystal was improved (5). Such improved dissolution of carbamazepine was attributed to the increased aqueous solubility by presence of excess glutaric acid, which minimized the precipitation of carbamazepine during dissolution. Dalsania et al. examined the impact of drug-polymer miscibility on enthalpy relaxation of Irbesartan ASDs (6). Results from this study suggests that drug-polymer miscibility determined at temperatures higher than the storage temperature should be viewed cautiously. Lubach and Hau utilized solid state nuclear magnetic resonance spectroscopy (NMR) to examine drug-excipient interactions and phase behavior in indomethacin-Eudragit $\mathrm{E}$ ASDs $(7) .{ }^{13} \mathrm{C}$ and ${ }^{15} \mathrm{~N}$ solid-state NMRs were used to determine changes in local structure and protonation state, while ${ }^{1} \mathrm{H} \mathrm{T}_{1}$ and $\mathrm{T}_{1 \rho}$ relaxation measurements were used to probe miscibility and phase behavior of the dispersions. This study highlights the prowess of using ss-NMR to understand solidstate properties of ASDs. Also from a fundamental perspective, Kalra et al. explored molecular speciation and crystallization mechanism of amorphous 2-phenylamino nicotinic 
acid using both spectroscopic measurements and computational analysis (8). The key finding of this study is that the primary intermolecular interaction could vary in different physical states affecting the phase transition kinetics.

For manufacturing ASD products, melt extrusion and spray drying techniques have been extensively employed in the pharmaceutical industry. In this issue, Chen et al. showed the polymer and drug were heterogeneous on the surface of spray dried ASD particles, which may lead to stability concerns (9). Another study by Teerakapibal et al. indicated surface coating of amorphous indomethacin with gelatin inhibited its rapid surface crystallization (10). We also have two innovative studies reported on melt extrusion. Wlodarski et al. designed a novel ternary melt extruded ASD system containing itraconazole, polyvinyl alcohol and copovidone (11). Such ternary system achieved both high solubilizing capability and superior precipitation inhibition. In another study, Arnfast et al. reported hot melt extrusion of indomethacin-cimetidine mixtures with $5 \%$ polyethylene oxide $(\mathrm{PEO})$ is a feasible way to manufacture ASD with minimum use of polymer excipients, while maintain sufficient physical stability (12).

Biopharmaceutical products including protein therapeutics have been increasingly developed in the past decade. Jiang et al. developed a multiparticulate system for colonic delivery of bovine serum albumin (BSA) (13). In this study, BSA was spray layered onto beads and coated using an enteric polymer EUDRAGIT® FS 30 D. Results showed BSA beads were protected from acidic media while BSA was released in pH 7.4 phosphate buffer, which demonstrated the feasibility to develop colonic delivery systems for proteins. Moussa et al. examined the effects of drying processes (lyophilization vs. spray drying) and excipients (mannitol, sucrose or trehalose) on the solid-state structural properties and potential stability (14). Traditional solid-state Fourier Transform Infrared Spectroscopy (ss-FTIR), solid-state fluorescence spectroscopy and relatively new method of solid-state hydrogen deuterium exchange with mass spectrometric analysis (ssHDX-MS) were employed in this study. Additionally, Liechty et al. investigated temporal evolution of the process gas during lyophilization using residual gas analysis technique (15). The residual gas analysis is able to detect oxygen and water vapor content in the process gas, which directly influence product shelf life.

We have two articles discussing particle engineering for developing high-dose dry powder inhalers (DPIs) to treat respiratory infections. Requirement of high-dose medications makes development of DPIs very challenging because use of excipients is limited to avoid high powder load. Mangal et al. developed a composite particle formulation of azithromycin using the spray drying technique (16). Incorporation of Lleucine in the composite particle significantly improved dissolution behavior of poorly water soluble azithromycin, which has great potential to improve treatment efficacy because azithromycin has to be dissolved to be active. In addition, Shetty et al. demonstrated that spray-dried amorphous ciprofloxacin DPI formulations tended to crystallize when they were stored at relative humidity $(\mathrm{RH})$ of $55 \%$ and higher (17). Such storage-induced crystallization led to an increase in aerosol performance as quantified by fine particle fraction (FPF).

Lastly, an article by Hiew et al. discusses the role of physico-chemical properties of excipients on the stability and formulation performance for pharmaceutical solid dosage forms (18). The report demonstrates the use of moisture sorption isotherms and dielectric spectroscopy to examine the effects of moisture on relaxation of two pharmaceutical excipients, starch and sodium starch glycolate, offering a clearer picture on how hydration impacts molecular relaxation in solids using moisture sorption isotherms and dielectric spectroscopy.

As the guest editors, we are pleased to have this special issue published with an impressing number of contributions. We thank the authors and we hope the readers will also find the articles insightful. Clearly, better drug products require fundamental understandings of materials properties and molecular dynamics through the formulation and manufacturing process; And this special issue showcases the interest.

\section{REFERENCES}

1. Xu T, Nahar K, Dave R, Bates S, Morris K. Polymorphic transformation of indomethacin during hot melt extrusion granulation: process and dissolution control. Pharm Res. 2018;35(7):140.

2. Koranne S, Thakral S, Suryanarayanan R. Effect of formulation and process parameters on the disproportionation of indomethacin sodium in buffered lyophilized formulations. Pharm Res. 2018;35(1):21.

3. Tinmanee R, Stamatis SD, Ueyama E, Morris KR, Kirsch LE. Polymorphic and covalent transformations of gabapentin in binary excipient mixtures after milling-induced stress. Pharm Res. 2018;35(2):39.

4. Hadjittofis E, Isbell MA, Karde V, Varghese S, Ghoroi C, Heng JY. Influences of crystal anisotropy in pharmaceutical process development. Pharm Res. 2018;35(5):100.

5. Van den Mooter G. The use of amorphous solid dispersions: a formulation strategy to overcome poor solubility and dissolution rate. Drug Discov Today Technol. 2012;9(2):e79-85.

6. Dalsania S, Sharma J, Munjal B, Bansal AK. Impact of drugpolymer miscibility on enthalpy relaxation of Irbesartan amorphous solid dispersions. Pharm Res. 2018;35(2):29.

7. Lubach JW, Hau J. Solid-state NMR investigation of drug-excipient interactions and phase behavior in indomethacin-Eudragit $\mathrm{E}$ amorphous solid dispersions. Pharm Res. 2018;35(3):65.

8. Kalra A, Lubach JW, Munson EJ, Li T. Exploring molecular speciation and crystallization mechanism of amorphous 2Phenylamino nicotinic acid. Pharm Res. 2018;35(3):51.

9. Chen Z, Yang K, Huang C, Zhu A, Yu L, Qian F. Surface enrichment and depletion of the active ingredient in spray dried amorphous solid dispersions. Pharm Res. 2018;35(2):38. 
10. Teerakapibal R, Gui Y, Yu L. Gelatin Nano-coating for inhibiting surface crystallization of amorphous drugs. Pharm Res. 2018;35(1):23.

11. Wlodarski K, Zhang F, Liu T, Sawicki W, Kipping T. Synergistic effect of polyvinyl alcohol and Copovidone in Itraconazole amorphous solid dispersions. Pharm Res. 2018;35(1):16.

12. Arnfast L, Kamruzzaman M, Löbmann K, Aho J, Baldursdottir S, Rades T, et al. Melt extrusion of high-dose co-amorphous drugdrug combinations. Pharm Res. 2017;34(12):2689-97.

13. Jiang B, Yu H, Zhang Y, Feng H, Hoag SW. A multiparticulate delivery system for potential colonic targeting using bovine serum albumin as a model protein. Pharm Res. 2017;34(12):2663-74.

14. Moussa EM, Wilson NE, Zhou QT, Singh SK, Nema S, Topp EM. Effects of drying process on an IgG1 monoclonal antibody using solid-state hydrogen deuterium exchange with mass spectrometric analysis (ssHDX-MS). Pharm Res. 2018;35(1):12.
15. Liechty ET, Strongrich AD, Moussa EM, Topp E, Alexeenko AA. In-situ molecular vapor composition measurements during Lyophilization. Pharm Res. 2018;35(6):115.

16. Mangal S, Nie H, Xu R, Guo R, Cavallaro A, Zemlyanov D, et al. Physico-chemical properties, aerosolization and dissolution of cospray dried azithromycin particles with L-leucine for inhalation. Pharm Res. 2018;35(2):28.

17. Shetty N, Zeng L, Mangal S, Nie H, Rowles MR, Guo R, et al. Effects of moisture-induced crystallization on the aerosol performance of spray dried amorphous ciprofloxacin powder formulations. Pharm Res. 2018;35(1):7.

18. Hiew TN, Huang R, Popov I, Feldman Y, Heng PWS. A study of moisture sorption and dielectric processes of starch and sodium starch glycolate. Pharm Res. 2017;34(12): 2675-88. 